\title{
REFLEXIONES TEÓRICAS CONTEMPORÁNEAS SOBRE PATRIMONIO EDIFICADO Y SU SIGNIFICADO ${ }^{(*)}$
}

\author{
CONTEMPORARY THEORETICAL REFLECTIONS ON BUILT HERITAGE AND ITS SIGNIFICANCE
}

\author{
TERESA VILLAMÓN GUEVARA ${ }^{[\star]}$ \\ Fecha de recepción: 22 de abril de 2017 \\ Fecha de aprobación: 15 de agosto de 2017
}

\begin{abstract}
RESUMEN
El patrimonio edificado, para ser reconocido formalmente como tal por instituciones nacionales y/o entes internacionales, se evalúa en el marco de una escala de valores históricos, estéticos y tecnológicos. Se aspira, a partir de ese reconocimiento, que la gestión de dicho patrimonio contribuya a la formación de una identidad cultural a nivel nacional o regional, la cual se ajuste a los ideales de las instituciones o comunidades que legitiman tal reconocimiento. No obstante, a nivel local o doméstico, los usuarios de ese patrimonio, si bien pueden reconocer esos valores, suelen atribuir al mismo objeto arquitectónico cualidades adicionales que se relacionan con su propia identidad cultural a un nivel de significado más íntimo, en una escala de carácter informal. A continuación, se presenta desde un enfoque interdisciplinario el debate académico contemporáneo sobre el significado del patrimonio, y los valores que este adquiere entre los grupos de actores formales -instituciones y profesionales del rubro- y ciudadanos, así como las tensiones y dicotomías que la naturaleza misma del patrimonio edificado genera entre quienes están vinculados a él.
\end{abstract}

\section{PALABRAS CLAVE}

Patrimonio edificado, valores culturales, recurso cultural

\section{ABSTRACT}

So that it may be formally recognized as such by national institutions and /or international bodies, built heritage is evaluated in a scale of historical, aesthetical and technological value. With such recognition as a starting point, the goal is to contribute to the formation of a cultural identity in a national or a regional scale through the management of the above-mentioned heritage. This identity would fit into the ideals of the institutions or communities that legitimize such recognition. Nevertheless, in a local or domestic level, even though users of this heritage can recognize such values in an architectural object, these very users tend to attribute to that same object, in a scale of informal character, qualities that relate to a level of more intimate meaning. The following essay presents the contemporary academic discussion around the concept of heritage, and the different values it acquires among groups of formal actors -institutions and professionals from the field-and citizens. As well, it shows the tensions and dichotomies that the nature of built heritage itself produces among those who are related to it.

\section{KEYWORDS}

Built heritage, cultural values, cultural resource

(*) El origen del presente artículo es fue extraído del marco teórico elaborado para la tesis de maestría de investigación titulada Valores culturales y conservación del patrimonio edificado en el centro histórico de Lima, destinada al programa de Maestría en Estudios Latinoamericanos del Centro de Documentación y Estudios Latinoamericanos (CEDLA) de la Universidad de Amsterdam, entre los años 2013 y 2014, con la supervisión de la doctora Christien Klaufus.

(**) Arquitecta titulada por la Universidad Ricardo Palma (2008), cuenta con el grado de magíster en Estudios Latinoamericanos por la Universidad de Amsterdam (2014). Su experiencia profesional en el sector privado incluye el diseño arquitectónico y la docencia universitaria, y en el sector público el desempeño de labores en la Dirección de Patrimonio Histórico del Ministerio de Cultura. Actualmente es docente de los cursos de Historia y Teoría de la Arquitectura 2, y Taller de Diseño 3 de la carrera de Arquitectura de la Universidad de Lima. Contacto: teresa.villamon@gmail.com 
El objetivo principal de este artículo es abordar el concepto de patrimonio cultural edificado, a fin de entender según qué valores o cualidades se le atribuye tal denominación y qué actores están involucrados en dicha atribución. Para ello, antes de adjudicarle un valor intrínseco, se lleva a cabo una revisión del debate académico. Esto, para mantener una perspectiva real de lo que puede ser importante para una sociedad y evitar caer en extremos conservacionistas o, por el contrario, en posiciones que fomenten el reemplazo del patrimonio por nuevas edificaciones, ignorando los valores que este ostenta. Así, en las siguientes líneas se revisa qué es lo que entendemos como patrimonio en sí mismo, quién o quiénes determinan su valor y, en consecuencia, cuáles son las razones por las que sería importante su preservación. Además, con el propósito de tener un mejor entendimiento de lo que significa el patrimonio edificado, se emplean ejemplos que permiten hacer evidente el rol del patrimonio arquitectónico en las ciudades contemporáneas, así como en los sitios arqueológicos. Adicionalmente, la relación entre identidad, ciudadanía y patrimonio arquitectónico se analiza de manera continua a través del texto. La discusión académica expuesta en este trabajo recoge diferentes enfoques, desde la experiencia de la Arqueología, la Antropología, la planificación urbana, la Geografía, la Historia del Arte y la conservación, tanto en Latinoamérica, como en Estados Unidos, España y el Reino Unido, debido a que el concepto patrimonio requiere un acercamiento multidisciplinario para definirse y ser manejado en la práctica.

El desarrollo del presente artículo está estructurado en dos secciones principales: "Enfoques sobre Patrimonio" y "Reconocimiento del Patrimonio". En la primera sección, se recogen ideas sobre el significado y usos del patrimonio de autores que lo relacionan con otros conceptos o lo abordan desde varias disciplinas. Al mismo tiempo, esta sección está dividida en dos partes, de acuerdo a los dos enfoques más utilizados para lidiar con el patrimonio: "De Patrimonio como Pasado a Práctica Social" y "Patrimonio como Recurso".

Así, la primera parte, "De Patrimonio como Pasado a Práctica Social", cuestiona la idea generalizada que considera el patrimonio como algo heredado de las generaciones pasadas por la sociedad presente. Insistir en esta "nostalgia" (Smith, 2006) puede, en ciertos casos, generar que se considere que el patrimonio debe ser preservado de manera estática, lo cual lo convierte en un obstáculo para la continuidad social y afecta así su posibilidad de evolucionar en el tiempo, tal como el resto de la sociedad. Desde otra perspectiva, sin embargo, si se toma como ejemplo el manejo de algunos sitios arqueológicos, resulta evidente que el patrimonio aún juega un rol activo en la producción de relaciones sociales (Breglia, 2006) y representa, a su vez, parte de la vida cotidiana de las personas que viven o trabajan en las áreas aledañas. Así, el patrimonio, en tanto elemento relacionado al pasado, comienza a adquirir otros significados, tales como práctica social', asociación de múltiples sujetos ${ }^{2}$ (Breglia, 2006), proceso culturaß ${ }^{3}$, práctica cultural/ (Smith, 2006), y empieza a vincularse a otros conceptos y disciplinas, tales como identidad e historia, que aportan una perspectiva del campo de influencia del concepto de patrimonio. En la segunda parte, titulada "Patrimonio como Recurso", se recogen argumentos de diferentes académicos que exponen la idea del patrimonio

\footnotetext{
1. Social practice (Breglia, 2006, p. 32)

2. Entendida como assemblage: "In archaeological parlance, an assemblage is a group of artifacts representing a culture.... my conceptualization of the heritage assemblage accounts for social actors, institutions deeply involved in both the production and reproduction of heritage" (Breglia, 2006, p. 11).

3. Cultural process (Smith, 2006, p. 44)

4. Cultural practices (Smith, 2006, p. 56)
} 
como una fuente rentable, susceptible de ser considerada para el desarrollo del turismo y, en consecuencia, plantean la necesidad de su inclusión en el planeamiento urbano.

Este trabajo aspira, además, a fomentar la reflexión sobre las diferentes escalas de valores respecto del patrimonio, sin separarlos de los recursos culturales de la vida cotidiana de los ciudadanos, quienes en la mayoría de los casos son sus consumidores y usuarios más frecuentes. Así, en la segunda sección, "Reconocimiento del Patrimonio", se presenta la discusión académica sobre la permanente coexistencia de dos -o másposiciones o valoraciones culturales respecto del patrimonio cultural. Esta dicotomía y tensión puede identificarse entre puntos de vista o actores formales (tales como instituciones y profesionales relacionados a la gestión del patrimonio), e informales (usuarios o ciudadanos). Asimismo, es posible encontrar otras ambivalencias, dependiendo del enfoque que se utilice para el análisis. Así, es posible referirse a una identidad nacional o una local, lo que Scarpacci (2005) Ilama "alto" y "bajo" capital cultural, o las macro y microactivaciones de patrimonio consideradas por Prats (2007).

\section{Enfoques sobre patrimonio}

La mayor parte del patrimonio formalmente reconocido está relacionado a sociedades anteriores. No obstante, en los párrafos siguientes se exponen enfoques alternativos en los que el patrimonio edificado adquiere un significado más amplio que su común asociación con el pasado. Entre ellos, que el patrimonio edificado puede ser también considerado como un recurso para obtener lucro económico, para la promoción de la cultura o para ambos; en esta sección, los enfoques académicos son presentados tomando en consideración ambas líneas de discusión. Al mismo tiempo, es posible encontrar que estas líneas de discusión se asocian a diversas áreas de estudio, lo que hace que el debate acerca de qué, por qué y para quiénes es el patrimonio implique un enfoque interdisciplinario.

\section{De patrimonio como pasado a práctica social}

El patrimonio está comúnmente asociado a sitios, lugares y artefactos antiguos, grandes, monumentales, y estéticamente placenteros (Smith, 2006). Esta asociación de ideas respecto a lo que se entiende como patrimonio está, según el autor, vinculada a la utilización de un factor de nostalgia por parte de la industria del patrimonio cultural, el cual finalmente se convierte en una herramienta del mismo sector para sustentar su posición sobre la gestión del patrimonio. Para el mismo autor, el riesgo de estos argumentos es que se traducen en una posición conservadora que obstaculiza la continuidad social y puede encasillar al público que esté de acuerdo con este mensaje como receptores pasivos del significado autorizado del patrimonio. Respecto de este punto, Breglia (2006) señala que:

While the term heritage readily evokes the past, it often obscures the everyday reality, the archaeological zones are active, dynamic, contingent spaces of the production of social relations....we can also think of heritage as a particular kind of social relationship, a postmodern search for origins, if you like, that references -without being predicated upon- material culture. (pp. 7-11)

Entonces, si se entiende el patrimonio más allá de datos arqueológicos o textos históricos (Smith, 2006), y además se toma en cuenta que es "el patrimonio de alguien"y que su experimentación lo convierte en algo vivo, resulta que este, como lo considera Breglia (2006), puede ser entendido como una práctica social, que en el uso se convierte en un recurso infinitamente renovable.

Es así que tanto Breglia (2006) como Smith (2006) concuerdan en considerar al patrimonio no solo desde su dimensión física, sino como un elemento dinámico dentro de la sociedad. Adicionalmente, Breglia toma de la arqueología el concepto de assem- 
blage o ensamblaje, para entender el patrimonio como una asociación de múltiples elementos, que incluyen actores sociales, paisaje, e instituciones involucradas en la producción y reproducción del mismo.

Tomando distancia de la calificación de patrimonio como un objeto, artefacto o lugar, incluso en los casos en que los aspectos tangibles juegan un papel importante (Smith, 2006), además del entendimiento del patrimonio como práctica social (Breglia, 2006), Smith (2006) propone entenderlo como un proceso cultural. Este proceso cultural involucra actos de memoria para facilitar el entendimiento y la relación con el presente. Es durante tales actos de memoria que los elementos tangibles asociados a un patrimonio determinado se convierten en herramientas culturales, aunque no son necesariamente indispensables para llevar a cabo dichos los de memoria que conforman el proceso cultural. Esto lo explica la autora a través del caso de la grabación de historias y tradiciones orales de las mujeres del pueblo waanyi en Australia: las mismas mujeres solicitaron ser grabadas narrando las historias en lugares culturalmente significativos de su propio territorio cultural: en ese caso, cada sitio patrimonial se convirtió en una ayuda-memoria o aide-mémoire para la trasmisión del conocimiento. En ese caso, "heritage was not the site itself, but the act of passing on knowledge in the culturally correct or appropriate contexts and times" (Smith, 2006, p. 46).

Es así que, si se considera que el patrimonio, sea tangible o intangible, es portador de valores y significados culturales, es necesario que dicho patrimonio sea experimentado para asegurar la incorporación, permanencia y vigencia de dichos valores en la identidad cultural de los usuarios. Así, una vez que el patrimonio puede ser experimentado, constituye también una práctica cultural o hábito involucrado en la construcción, y la regulación de un rango de valores y conocimientos. Es entonces que, en ese contexto, el patrimonio puede también ser interpretado como identidad que, tal como la historia, promueve el sentido de pertenencia y continuidad de un grupo de personas.

Sin embargo, según Smith (2006) existe un discurso hegemónico alrededor del patrimonio que podría dejar de lado el encuentro de maneras alternativas, acordes a cada realidad, para identificar y gestionar el patrimonio:

The "heritage" discourse therefore naturalizes the practice of rounding up the usual suspects to conserve and "pass on" to future generation, and in so doing promotes a certain set of Western elite cultural values as being universally applicable. Consequently, this discourse validates a set of practices and performances, which populates both popular and expert constructions of "heritage" and undermines alternative and subaltern ideas about "heritage". (p. 11)

No obstante, a propósito de lo afirmado por Smith (2006), cabe afirmar que, a diferencia de lo que sucede en las culturas occidentales, en algunas culturas orientales se da mayor valor al conocimiento que permite continuar produciendo determinado tipo de construcción, como en el caso del templo Shinto de Ise en Japón, el cual se renueva hace más de 20 siglos utilizando la misma técnica de trabajo en madera (Vecco, 2010). Más allá de la conservación del material, es el espíritu del edificio identificado en la transmisión de un conocimiento técnico lo que cobra mayor importancia. Desde una perspectiva similar, en 1993, la República de Corea planteó a la Organización de las Naciones Unidas para la Educación, la Ciencia y la Cultura (UNESCO) la creación de un plan de Tesoros Humanos Vivos, asociado a la transmisión oral del patrimonio inmaterial. En ese sentido, cabe mencionar que el texto de la Convención Para la Salvaguardia del Patrimonio Cultural Inmaterial (UNESCO, 2003) considera la interdependencia entre el patrimonio cultural inmaterial, y el patrimonio cultural y natural.

En cuanto a la búsqueda de enfoques del patrimonio, además de su común relación con el pasado, se encuentra la propuesta de Howard (2003) de considerar el patrimonio en sí mismo como una disciplina aplicada: "Heritage is not about the past. Of course, many of the objects and the ideas with which it deals come from the past, but heritage 
issues are always about what we do with them now" (p. 19). Con esta afirmación, coincide con la visión de Smith (2006) de intentar buscar un enfoque que considere al patrimonio un elemento que forma parte de la sociedad contemporánea, y no lo reduzca a un elemento del pasado, desconectado del momento actual. Sin embargo, Howard va más allá, considerando el patrimonio una disciplina aplicada en sí misma (2003), la que como tal involucra el aspecto teórico del concepto de patrimonio desde distintas disciplinas que de manera directa o tangencial se ocupan de su estudio de forma que, desde el conocimiento integrado del mismo, sea posible brindar directrices para su manejo. Al respecto de la necesidad de hacer del patrimonio una disciplina práctica, al hacer un análisis del vínculo entre la Historia y el patrimonio, Howard hace énfasis en el hecho de que una cantidad considerable de objetos patrimoniales despiertan poco o nada de interés entre los historiadores (2003, p. 21). En su argumento, el autor considera que la Historia es un humanismo puro, interesado en el pasado, mientras que los estudios acerca del patrimonio se plantean cómo ese pasado puede ser interpretado para ser utilizado en beneficio de las sociedades actuales.

En el caso de la Geografía y la Historia del Arte, Howard (2003) también resalta que son las dos disciplinas desde las que más interés se ha mostrado en el tema del patrimonio. Esto, según él, sucede en parte porque la geografía comparte tres puntos comunes con la gestión del patrimonio: (a) el interés en la combinación de los materiales naturales y culturales, (b) sus aplicaciones prácticas en la resolución de las necesidades de la sociedad actual y, finalmente, (c) la dificultad de ambas de delimitar sus campos de estudio. En el caso de la Historia del Arte, a pesar de incluir el estudio del significado de los objetos (semiología), los estudios de patrimonio también están relacionados a cómo garantizar su preservación.

\section{Patrimonio como recurso}

Breglia (2006) y Howard (2003) coinciden en considerar el patrimonio como un recurso renovable, y al respecto plantean como principal interrogante cómo debe ser utilizado. Así, el segundo hace énfasis en el trabajo multidisciplinario que implica la gestión del patrimonio, y, a propósito de la conexión entre patrimonio, y el ocio y el turismo, señala que muchas publicaciones sobre patrimonio son realizadas por académicos que trabajan en ocio y turismo. Esta relación entre el patrimonio y la industria turística ha facilitado que el primero sea colocado en las agendas de muchos gobiernos como una actividad potencialmente lucrativa. Sin embargo, para Howard (2003), el patrimonio "is not only for tourists, it is also for pilgrims, for insiders, for members of the family who never leave home..., and it is for academics and for governments of many levels" (p. 25). Es así que para los turistas el sentido de pertenencia al experimentar el patrimonio no es el mismo que la experiencia de los habitantes locales. Sin embargo, no se debe olvidar que la relación entre el turismo y el patrimonio no es reciente; de hecho, este último sigue siendo una de las principales razones para adquirir paquetes turísticos (Prats, 2009). No obstante, el turismo masivo y la espectacularización de la realidad producen un cambio cualitativo y cuantitativo en la relación entre el patrimonio y el turismo. Así, por ejemplo, a veces el crecimiento del mercado turístico afecta la conservación de un sitio patrimonial por el uso intensivo que se le da para satisfacer sus requerimientos. Al mismo tiempo, la necesidad de satisfacer la demanda turística también contribuye a abrir otros lugares patrimoniales "menos importantes" (Prats 2009, p. 41). En este contexto, Scarpacci (2005) señala otros inconvenientes que el turismo puede conllevar en áreas monumentales, tales como gentrificación y otras dificultades sociales. No obstante, el autor considera al patrimonio un recurso económico para el presente, y ve en el uso de las áreas históricas y monumentos una herramienta para construir la identidad nacional y forjar ideologías, aunque "the tension created over what is to be preserved, whose collective memory should be celebrated, is often ignored in official public circles" (2005, p. 16). Por lo tanto, debe hacerse énfasis en que el patrimonio sea manejado también para 
beneficio de la comunidad local, tomando en cuenta el significado particular que este recurso cultural tiene para ella.

Respecto a los centros históricos, Herzog (2006) argumenta que, mientras que por un lado se hace referencia al fin del espacio físico, por el otro, en la actualidad es importante tomar en cuenta los primeros como ejemplos de diseño urbano que consideran la escala peatonal, buscan la cohesión social de los miembros de esas comunidades y representan un determinado sentido del lugar. En este sentido, lo descrito por propone el uso del patrimonio edificado a modo de recurso para la formación de una identidad comunitaria y un modelo en cuanto a criterios para el diseño urbano, en un tipo de gestión donde el patrimonio edificado se considere más que un recurso económico a ser aprovechado a través del turismo.

En cuanto a la legislación, Scarpacci (2005) subraya que, por un lado, la Carta de Quito (1967) acercó entre sí las ideas sobre monumentos, legislación y planificación urbana; además, provocó una oleada de interés en los centros históricos latinoamericanos y fortaleció la preservación histórica en la región. Sin embargo, esto no significó la implementación de grandes proyectos de inversión en el campo. Cita a Gerald Greenfield, que antes de la segunda mitad del siglo XX afirmaba que "los estudiosos habían ignorado durante mucho tiempo la América Latina urbana porque la América española había sido considerada un reino agrario" (citado en Scarpacci, 2005, p. 29). También destaca que uno de los hechos que dificultan el logro de la Organización de las Naciones Unidas (ONU) en torno al turismo patrimonial (Carta de Quito) es el descontento, la apatía o la falta de tiempo por parte de los residentes de los centros históricos.

Finalmente, Prats (2009), que propone el entendimiento del patrimonio como construcción social, considera que este puede cambiar con el tiempo de acuerdo con nuevos criterios o intereses dados por nuevas circunstancias. Para el geógrafo e historiador, la naturaleza, la historia y la inspiración creativa son los tres factores clave mediante los cuales es posible encontrar símbolos patrimoniales que son potencialmente patrimonio. A la vez, estos símbolos patrimoniales son considerados como recursos, tanto culturales como turísticos, y activados -principalmente por las instituciones nacionales- sobre la base de versiones ideológicas de la identidad. Para Prats (2009), de modo similar al patrimonio, el concepto de identidad también puede ser considerado una construcción social y un hecho dinámico en el tiempo; así, el patrimonio es la representación simbólica de cualquier identidad.

\section{Reconocimiento del patrimonio}

Not everything is heritage, but anything could become heritage. (Howard, 2003, p. 7)

El reconocimiento del valor del patrimonio como tal es fundamental para su protección, ya sea a nivel nacional o a menor escala (Howard, 2003). Asimismo, el patrimonio formalmente reconocido no está imposibilitado de contener, al mismo tiempo, una identidad asociada a la definición de nación, un valor a nivel de comunidades o doméstica. En ese contexto, Howard toma como ejemplo la rubbish theory o the circuit of culture, "where artefacts become obsolescent and are categorized as useless rubbish, until they become revaluated and take their part in heritage developments" (2003, p. 46). Por su parte, las arqueólogas Breglia (2006) y Smith (2006) desarrollan ideas acerca del patrimonio tomando como referente sitios arqueológicos para analizar los diferentes actores y facetas alrededor de este concepto, mostrando, además, cómo cada uno de estos actores puede atribuir un valor particular al patrimonio, el cual no necesariamente es el mismo para cada actor. Por consiguiente, resulta que el valor de un mismo elemento patrimonial puede ser atribuido de manera diversa desde diferentes puntos de vista, dependiendo este último del sujeto o grupo humano que atribuye el valor que lo convierte en patrimonio (a nivel internacional, nacional, local o doméstico). 
Asimismo, respecto al reconocimiento del patrimonio como tal, en la literatura comúnmente se encuentra mención a un patrimonio considerado "formal", entendido como tal aquel que ha sido reconocido por instituciones nacionales o internacionales (Smith, 2006). Esta validación permite la implementación de políticas para su promoción y preservación. Por otro lado, existe un patrimonio "informal", considerado folklore, o que concierne a pequeñas comunidades, como barrios o familias (Howard, 2003). Este trabajo se centrará en el patrimonio formalmente reconocido.

\section{Valores formales e informales}

El valor de patrimonio, como lo plantea Breglia (2006), recae en la valoración que le atribuyen los diferentes actores involucrados en la gestión del patrimonio cultural. Al coexistir diversos intereses, como los del Estado, los residentes locales, los propietarios, los profesionales y los empresarios, tal gestión resulta complicada, pues las definiciones de la mejor manera de llevar a cabo la tarea frecuentemente son planteadas de forma opuesta desde las posturas formales (instituciones) e informales (habitantes). En este contexto, Breglia (2006) resalta la ambivalencia entre la posición del Estado, que emplea el patrimonio para la creación de una identidad nacional y sentido de pertenencia, y la manera en que la ciudadanía se relaciona con este.

Para analizar esta dualidad o múltiples posiciones en torno al patrimonio, Breglia (2006) basa su investigación en casos de sitios arqueológicos en México donde, según ella, coexisten múltiples reivindicaciones. Así, muestra cómo durante el gobierno de Salinas de Gortari el Estado inició un programa de privatización de sectores de servicios anteriormente nacionales. A pesar de la privatización de muchos sectores, en el caso del patrimonio cultural tal política no fue aceptada por varios grupos de intelectuales, periodistas y ciudadanos. Bajo el lema "Nuestro patrimonio cultural no está a la venta", después de manifestaciones de estos grupos contra la iniciativa de privatización, la propuesta estatal finalmente fue descartada en 1999: según la Constitución, el patrimonio mexicano sigue siendo propiedad nacional.

Además, Breglia (2006) analiza cómo el Estado mexicano ha manejado la relación entre la voluntad de preservar su patrimonio cultural, y el deseo de ser un estado moderno y neoliberal. Para ello toma dos estudios de caso: Chichén Itzá y Chunchucmil. Utilizando estos dos ejemplos, presenta únala ambivalencia existente entre los sitios arqueológicos, y los actores e instituciones sociales. En ambos casos, la autora muestra cómo en el caso de Chichén Itzá, a pesar de que se consideran oficialmente como sitios de propiedad nacional, existen grupos de habitantes que reclaman su custodia en tanto un privilegio heredado. Por otra parte, en el caso de Chunchucmil, un sitio arqueológico declarado recientemente -por extensión se ha nacionalizado-, los agricultores que anteriormente ocupaban esta tierra y sus alrededores tenían que lidiar con las tensiones creadas entre sus intereses y los de los arqueólogos. Acerca de esta ambivalencia, Breglia afirma:"Para las comunidades locales mayas, el patrimonio forma parte de una experiencia cotidiana y de conocimientos de sentido común. Las ruinas arqueológicas... forman parte del paisaje social, político, cultural y económico" (2006, p. 208).

Por su parte, Scarpacci (2005) se enfoca en nueve casos de cascos históricos en Latinoamérica, en Buenos Aires, Bogotá, Montevideo, Cartagena, Cuenca, La Habana, Puebla, Quito y Trinidad. Los últimos seis centros históricos están reconocidos como Patrimonio Cultural de la Humanidad por UNESCO. Aún cuando los métodos de Scarpacci incluyeron entrevistas con empleados del sector público en La Habana, Cartagena y Cuenca, su intención principal era conocer cómo vivían las personas en estas ciudades y cómo se había dado la evolución de sus áreas históricas. Además, el geógrafo planteó preguntas sobre cómo los habitantes se sentían en relación al turismo internacional, y los planificadores y políticos, a fin de determinar si se sentían parte de la mejora de sus centros históricos o, por el contrario, se sentían excluidos del proceso. Tal como Breglia (2006), Scarpacci (2005) también encontró diferentes escalas de valo- 
res respecto al patrimonio, las que llama capital cultural "alto" y "bajo". Así, identifica las plazas y edificios principales capital cultural alto, y considera capital cultural bajo a la arquitectura vernacular y los espacios públicos secundarios. El capital cultural bajo pocas veces aparece en las guías de turismo, sitios de Internet y publicidad, aun cuando es parte importante de la vida del centro de las ciudades.

El discurso patrimonial, para Smith (2006), se basa en un conjunto de valores y significados que tienen una voz autorizada en los profesionales, los Estados y las instituciones internacionales. En este discurso de patrimonio autorizado, las relaciones de poder se establecen alrededor del patrimonio, teniendo autoridad para hablar, o para el patrimonio y los que no lo hacen (p. 12). Sin embargo, es evidente que a través de documentos como la Declaración Universal Sobre la Diversidad Cultural (UNESCO, 2001), se entiende que las identidades culturales son valoradas desde su pluralidad, variedad y dinamismo, sin duda en un esfuerzo de las instituciones internacionales por actualizar su discurso para que sea suficientemente versátil frente a la diversidad cultural de las sociedades.

\section{Escalas de patrimonio}

Llorenc Prats (2009) también diferencia dos posiciones en torno a la definición del patrimonio, en la forma de dos tipos de activaciones. Las primeras son aquellas promovidas, financiadas y manejadas a escala macro, usualmente por el Estado. Las segundas son aquellas activaciones a escala micro, de iniciativa local y algunas veces apoyadas por los gobiernos locales. De acuerdo con Prats (2009), las iniciativas macro usualmente tienen objetivos a gran escala, frecuentemente dirigidos a atraer masas, tales como la apertura de museos y parques nacionales. Por el contrario, las activaciones micro están más enfocadas en la subsistencia, y tienen un moderado impacto en la economía y la dinámica sociocultural locales.

Ahora bien, lo que Breglia (2006) define como ambivalencia, en referencia a los diferentes intereses y significados sobre lo que es patrimonio según cada uno de los actores, puede estar estrechamente relacionado a lo que Prats (2009) define como macro y microactivaciones, o escalas de iniciativas a nivel local o nacional. Asimismo, se puede establecer una relación similar con lo que Howard (2003) define como dos tipos de patrimonio: nacional y familiar. El primero, institucionalizado, es tomado como la representación de la identidad nacional, donde las políticas de gestión del mismo -implementadas por las instituciones del Estado- apuntan a que este patrimonio sea sentido como propio por toda la población. El segundo tipo de patrimonio, familiar, se refiere a recuerdos de familia y objetos de valor no oficial, en los que, según Howard (2003), la mayoría de personas tiende a pensar cuando son preguntados acerca de qué le quieren dejar a sus descendientes.

A pesar de la discusión que resulta acerca de las diferentes posiciones respecto de lo que el patrimonio representa en diferentes esferas, a través del tiempo, los gobiernos y el mercado son los que han definido y promovido la preservación del patrimonio cultural, sobre la base de la definición institucionalizada del patrimonio. Sin embargo, ante esto, Gómez Ferri (2004) propone considerar el surgimiento de la sociedad civil como un tercer y nuevo agente que últimamente está tomando parte en este escenario. El profesor en Sociología y Antropología Social toma como ejemplos concretos asociaciones civiles propatrimonio organizadas en Valencia, España: Salvem el Botanic, Salvem el Cabanyal y Salvem l'Horta. Estas tres plataformas fueron organizadas por ciudadanos que reclamaban a las instituciones la preservación del jardín botánico, el barrio de El Cabanyal y la huerta de Valencia, respectivamente. En el trabajo de Gómez Ferri (2004), es posible reconocer dos dimensiones: (a) la dignificación de la identidad y la recuperación de la autoestima colectiva de los vecinos, y (b) la reactivación y el reconocimiento del valor del patrimonio que representa para ellos su vecindario, en lo que respecta a aspectos tangibles, como su arquitectura y diseño urbano, e intangibles, como historias y hechos recordados por los habitantes más antiguos de la zona. 


\section{Valores arquitectónicos y procesos psicológicos}

De manera similar al planteamiento de Gómez Ferri (2004), McCarthy (2012) se dirige también a la contradicción entre lo que los profesionales del patrimonio y los desarrolladores inmobiliarios reconocen como tal, y la percepción de este valor que le atribuye la población. Sin embargo, McCarty aporta desde otra perspectiva, tomando teorías psicológicas de la percepción del riesgo y sugiriendo que podrían ser relevantes para proponer nuevas estrategias en cuanto a la gestión del patrimonio. Se refiere a algunas particularidades que implican la preservación del patrimonio construido, afirmando que la estructura física de un edificio también puede incluir la historia, las ideas, los recuerdos y el significado. Asimismo, en el caso particular de la arquitectura, la gestión del patrimonio lidia también con el hecho de la necesidad de ser experimentado físicamente, en escala y tres dimensiones: "While documentation can mitigate some aspects of loss, the scale and three-dimensional experience of aspects of architectural heritage limit the viability of two-dimensional recording as a substitute for physical buildings" (McCarthy, 2012, p. 625). La experiencia tridimensional a la que se refiere McCarthy no solo incluye la arquitectura, sino que también la arqueología se ocupa de este desafío:

This is not to advocate a black-and-white world where everything must be preserved, nor to contradict recent debate asserting heritage's intangibility (Smith, 2006), but rather to indicate there is a value in the preservation of the built fabric of buildings, as well as of other manifestations of heritage. Such issues are particularly relevant for heritage architecture because of capitalist modes of ownership, where structures that manifest communal heritage are exclusively owned. (2012, p. 625)

Por otra parte, McCarthy (2012) considera que el término edificio patrimonial no significa necesariamente que esté declarado oficialmente como tal, sino que refleja los valores heredados de una comunidad. Estos valores y significados están detrás del surgimiento de algunas protestas comunitarias contra la demolición de edificios que simbolizan una parte de su propia identidad. Sin embargo, la mayoría de estas protestas se organizan cuando las autoridades ya han concedido permisos para modificar o eliminar un edificio patrimonial, lo que parece un hecho contradictorio que aparentemente aparece en situaciones extremas. Aquí es donde McCarthy propone analizar el significado del patrimonio y su preservación teniendo en cuenta la percepción del riesgo, para encontrar por qué esta contradicción sucede. Según la percepción del riesgo -asumiendo que el riesgo es la pérdida de patrimonio-, está vinculado con el tiempo de descuento, y el temor y peligro de lo desconocido. En cuanto al primero, McCarthy toma el término de Weber en el que descontar el tiempo significa que las personas preferirán una recompensa menor si reciben un beneficio más pronto por sobre una recompensa o beneficio mayor que se recibe más tarde (2012, p. 628). Por otra parte, temor y riesgo de lo desconocido son términos tomados de la obra de Slovic, que sugieren que lo conocido y que no inspira temor no es percibido como riesgo significativo. Ello explicaría la dejadez en algunos casos frente a la posibilidad de la pérdida de patrimonio, especialmente si la comparamos con el temor hacia hechos percibidos como más urgentes, como puede ser la prevención de enfermedades o las consecuencias de eventos meteorológicos, porque existe una distancia temporal involucrada:

Risk, which is immediately knowable and viscerally felt, will be prepared for, but heritage protection frequently relies on the cognitive (rather that effective), making motivation and risk preparation less likely, because heritage protection asks for an action to prevent an event in the distant future (the destruction of a heritage building) which may or may not happen (McCarthy, 2012, p. 627).

McCarthy (2012) también se refiere a la paradoja entre la idea de preservar el patrimonio, lo cual parece positivo para la mayoría de la gente, y la idea de poseer un edificio patrimonial, lo que se percibe generalmente como una situación que podría afectar 
negativamente a sus derechos de propiedad. No obstante, para el autor este aspecto no estaría relacionado con una distancia psicológica, sino con un conflicto entre propiedad común y propiedad privada.

\section{Reflexión final}

Tal como hemos visto, la discusión académica alrededor del concepto de patrimonio se plantea desde distintos enfoques, dependiendo en parte de la propia experiencia profesional del autor. Así, mientras Breglia (2006) y Smith (2006) toman como referentes sitios arqueológicos, Howard (2003) intenta dar una visión más amplia de cómo el patrimonio está relacionado no solo con otras disciplinas, sino que el patrimonio puede ser entendido desde diferentes puntos de vista. Resulta evidente, entonces, que no hay una única manera de determinar una definición universal de patrimonio, sino que el significado de este depende del enfoque desde el cual se quiera abordar el concepto. Así, Breglia (2006), Smith (2006), Prats (2009), McCarthy (2011), Herzog (2006), Scarpacci (2005) y Howard (2003) podrían coincidir en que pueden existir múltiples interpretaciones, dependiendo de quién define el valor del patrimonio cultural. Las contradicciones entre las políticas gubernamentales en cuanto al manejo de sitios arqueológicos y la posición de las poblaciones nativas que habitan en sus alrededores, a las que Breglia (2006) se refiere como monumental ambivalence, pueden interpretarse también como las tensiones creadas entre los proyectos oficiales y la sociedad civil, las mismas que llevaron a la segunda a organizar grupos como Salvem el Cabanyal (Gómez Ferri, 2004), con la diferencia de que en este caso la ambivalencia se situaba en un contexto urbano e implicaba patrimonio arquitectónico. Asimismo, estas posiciones, usualmente desconectadas, están también señaladas por Howard (2003), quien afirma que "Heritage is for people; not just for a small minority of specialists and experts, but for everyone" (p. 33).

Así, en el debate académico se encuentra que, en muchos sentidos, el patrimonio se constituye siempre en una dicotomía. El primero de estos sería el discurso oficial que evalúa qué símbolos de la cultura de un grupo son suficientemente representativos para ser considerados patrimonio. Este aspecto oficial del patrimonio cultural está en manos del Estado, profesionales y organizaciones internacionales como UNESCO o ICOMOS, que de acuerdo a una específica escala de valores declaran y sugieren la protección de ciertas demostraciones materiales e inmateriales. Por otro lado, se agrupa bajo la etiqueta de "folklor" o"artesanías"a otros aspectos que a nivel local podrían tener mayor significado para la población que el patrimonio nacional. Tomando en cuenta que las declaratorias de patrimonio formalmente reconocido están en manos del Estado -como es también el caso de Perú-, es hasta cierto punto lógico que se apunte a que estas evidencias culturales puedan llegar a representar a toda la nación. Más aún, las iniciativas nacionales están generalmente comprometidas en proyectos de gran escala, tal como Prats (2009) menciona, que apuntan a dar acceso masivo a esa cultura nacional. ${ }^{5}$ Sin embargo, esa "elitización", como puede ser visto el que un grupo de autoridades determine qué es valioso y qué no -o no tanto-, no quiere decir que a nivel local la población no se sienta representada en otros aspectos culturales que conforman, quizás, una escala de patrimonio más íntima relacionada al mismo artefacto, sitio o práctica.

En ese sentido, la identificación local con ciertas manifestaciones culturales puede generar, como plantea Gómez Ferri (2004) respecto del caso de El Cabanyal, el interés de un sector de la población en conservar ciertos espacios públicos o edificios, no necesariamente basándose en valores oficiales, como también recuerda McCarthy

\footnotetext{
5. Existen incluso ejemplos de coordinación entre varios gobiernos, como para el Proyecto Qhapaq Nan, a fin de promover la investigación, registro, conservación, puesta en valor y reconocimiento ante UNESCO como Patrimonio Cultural de la Humanidad, de la red de caminos inca. En este proyecto se involucran los gobiernos de Perú, Colombia, Ecuador, Bolivia, Chile y Argentina. El sitio web del proyecto es http://www. qhapaqnan.gob.pe/wordpress/.
} 
a manera personal: "One friend, emailing following the recent Christchurch earthquake [February 2011] and news of likely demolition of Knox Church, expressed her sorrow at the thought of no longer being able to show her children the building where she was married" (2011, p. 625).

Entonces, resulta que el patrimonio implica constantemente dos -o más- niveles de representación. Uno a escala nacional, utilizado para la construcción de la identidad nacional, en pro de la cohesión social; y escalas de significado locales e incluso domésticas.

Al mismo tiempo, la valoración oficial otorgada desde las instituciones a ciertas manifestaciones culturales, que les atribuye la categoría de patrimonio, puede generar otras dos escalas de valoración, tal como Scarpacci (2005) argumenta en su concepción de capital cultural alto y bajo. La definición de un capital cultural considerado de alto valor y otro de valor menor se puede relacionar directamente con la gestión del turismo cultural. Así, podría ser positivo tener esto en cuenta para promover el turismo hacia aquellos lugares culturales considerados bajos como una herramienta para reducir el impacto que conlleva la alta concentración turística en los lugares de valor cultural alto y, al mismo tiempo, promover la conservación de otros edificios y espacios públicos (Prats, 2009).

Finalmente, es posible afirmar que en cuanto a la construcción de la identidad nacional, tanto a nivel local y doméstico como para los turistas, el patrimonio cobra diferentes sentidos de pertenencia y valores. De ello deriva que Breglia (2006) y Howard (2003) coincidan en plantear el patrimonio como un recurso renovable, ante el que la principal cuestión es cómo gestionarlo. El reto que conlleva entonces la naturaleza multifacética del patrimonio es el de su gestión como recurso, entendiendo recurso en su sentido más amplio, como generador de relaciones sociales, de empleos o como medio para interpretar el presente, considerando además que ese manejo debe contemplar y respetar sus diferentes escalas de valoración: nacional, local o doméstica.

\section{Referencias}

Breglia, L. (2006). Monumental ambivalence: the politics of heritage. Austin: University of Texas Press.

Gómez Ferri, J. (2004). Del patrimonio a la identidad: la sociedad civil como activadora patrimonial en la ciudad de Valencia. Gazeta de Antropología, 20, (art. 9).

Herzog, L. A. (2006). Return to the center: culture, public space, and city-building in a global era. Austin: University of Texas Press.

Howard, P. (2003). Heritage: management, interpretation, identity. Londres, Reino Unido; y Nueva York: Continuum.

Prats, L. (2009). Antropología y patrimonio. Barcelona, España: Ariel.

McCarthy, C. (2012). Re-thinking threats to architectural heritage. International Journal of Heritage Studies, 18(6), 624-636.

Scarpaci, J. L. (2005). Plazas and barrios: heritage tourism and globalization in the Latin American centro histórico. Tucson: University of Arizona Press.

Smith, L. (2006). Uses of heritage. Londres, Reino Unido; y Nueva York: Routledge.

Vecco, M. (2010). A definition of cultural heritage: from the tangible to the intangible. Journal of Cultural Heritage, 11(3), 321-324. 